\title{
Mechanistic study of angiogenesis induced by shh-transfected BMMSC transplantation after myocardial infarction
}

\author{
Fenglin Song ${ }^{1}$, Ming $\mathrm{Wu}^{1,2}$, Tao $\mathrm{Tang}^{1}$, Xiaoming $\mathrm{Wu}^{1}$, Wenwu $\mathrm{Zhou}^{2}$, Jin $\mathrm{Shi}^{1}$, \\ Wei Zhang ${ }^{1}$, Zhiping Tan $^{1}$, Jinfu Yang ${ }^{1^{*}}$ \\ ${ }^{1}$ Department of the Cardiothoracic Surgery of the 2nd Xiangya Hosptial, Central South University, Changsha, China; \\ *Corresponding Author: yjf19682005@yahoo.com.cn \\ ${ }^{2}$ Department of the Cardiothoracic Surgery of the Hunan Provincial People's Hospital, Changsha, China
}

Received 25 February 2013; revised 25 March 2013; accepted 15 April 2013

Copyright (C) 2013 Fenglin Song et al. This is an open access article distributed under the Creative Commons Attribution License, which permits unrestricted use, distribution, and reproduction in any medium, provided the original work is properly cited.

\begin{abstract}
Objective: To investigate the changes and mechanism of angiogenesis in myocardium induced by transplantation of the sonic hedgehog (shh) gene transfected in bone marrow mesenchymal stem cells (BMMSC) after myocardial infarction. Methods: A rat model of acute myocardial infarction was made by coronary artery ligation. The rats were randomly divided into five groups of $\mathbf{4 0}$ rats each. These were further subdivided into groups of 10 rats. The peripheral regions of the infarcts were injected with either BMMSC $^{\mathrm{SHH}}$ (transfection group), equivalent BMMSC (cell only group), BMMSC and pcDNA3.1Shh DNA mixture (mixture group), pcDNA3.1shh DNA alone (gene only group), or equal volumes of low-sugar DMEM medium (control group). One, two, four, and eight weeks after transplantation, specimens were harvested from the transplantation site to determine the expression of SHH signaling pathway downstream genes Ptc1, Gli-2, COUP-TF II, angiogenesis promoting factor VEGF, and Ang-1 using RTPCR. Results: Seven days after transplantation, the expression of SHH signaling pathway downstream genes, Ptc1, Gli-2, and COUP-TF II was significantly more pronounced in the transfection group than in the control group, cell only group, gene only group, or mixture group (Ptc1: $P<0.01, P<0.01, P<0.05$, and $P<0.05$, respectively; COUP-TF II: $P<0.01, P<0.01, P<0.05$, and $P<0.05$, respectively; Gli-2: $P<0.01, P<$ $0.01, P<0.05$, and $P<0.05$, respectively). The expression of angiogenesis-promoting genes
\end{abstract}

Vegf and Ang-1 was significantly more pronounced than in the control group, cell only group, or gene only group (Vegf: $P<0.01, P<$ 0.05, $P<0.05$ respectively; Ang-1: $P<0.01, P<$ 0.05 , and $P<0.05$, respectively). Conclusion: Transplantation of the shh-gene-transfected BMMSCs to the peripheral regions of myocardial infarcts promoted angiogenesis by upregulating downstream gene expression.

Keywords: Shh; Bone Marrow Mesenchymal Stem Cells; Ptc1; Gli-2; COUP-TF II; VEGF; Ang-1

\section{INTRODUCTION}

The study of cells and genes that promote angiogenesis in the treatment of ischemic heart disease has become a new hotspot in life science research. Cell therapy improves cardiac function by transplanting stem cells from certain sources into the cardiomyopathic area and promoting their proliferation and differentiation into cardiomyocytes. This restores the number of functional cardiomyocytes. However, the survival rate of the transplanted cells is low due to the low blood perfusion of the ischemic area. Gene therapy improves cardiac function by introducing genes encoding an angiogenic growth factor into the body, promoting angiogenesis in the local ischemic area and improving blood supply to ischemic tissue. These two types of methods each have their own limitations, so combinations of cells and gene therapy are a major new direction of study in this field.

Bone marrow mesenchymal stem cells (BMMSC) are self-renewal pluripotent cells found in bone marrow. Under certain conditions, they can differentiate into a variety of functional cells, including cardiomyocyte-like 
cells [1-3]. In this way, they hold broad clinical application prospects for the treatment of ischemic heart disease. $\mathrm{SHH}$ is the most important member of the Hedgehog (Hh) gene group. These genes encode a secretory signaling protein. It is expressed widely during vertebrate development and can promote angiogenesis and cell growth, it is especially important in the development of the cardiovascular system in embryos. In mature animals, SHH can promote blood vessel growth by upregulating the joint expression of angiogenesis-promoting factors such as VEGF and Ang-1. SHH's ability to stimulate blood vessel growth is very strong. The newly formed blood vessels are larger, more mature, and have better functions [4-6]. Based on this, we transferred the SHH gene into BMMSCs and explored its angiogenic pathways and possible mechanisms to provide a theoretical basis for joint cell and gene therapy for ischemic heart diseases.

\section{MATERIAL AND METHODS}

\subsection{Experimental Animals and Main Materials}

Wistar rats were purchased from Shanghai Laboratory Animal Center, Chinese Academy of Sciences. All animals received humane care in compliance with the "Guide for the Care and Use of Laboratory Animals" prepared by the Institute of Laboratory Animal Resources, National Research Council, and published by the "Guide to the Care and Use of Experimental Animals" by the Chinese Council on Animal Care. DMEM lowglucose medium and Australia fetal bovine serum were obtained from Gibco. Lymphocyte separation medium was purchased from Sigma. Trizol was purchased from Genewindows. A reverse transcription kit was purchased from Fermentas. A qPCR kit was purchased from Applied Biosystems. Anti-Shh polyclonal rabbit antibodies were purchased from Proteintech. An odyssey bicolor infrared fluorescence imaging system was used for Western blotting. A transfection kit was purchased from Amaxa. PCR reagents were purchased from Fermentas. Primers were synthesized by Shanghai Sangon.

\subsection{Harvesting BMMSCs for Transfection}

The broken femurs of rats were washed with saline, and $1.073 \mathrm{~g} / \mathrm{ml}$ Percoll was used for preliminary separation of bone marrow mesenchymal stem cells (BMMSC). Then BMMSCs were further isolated and purified by replacing old medium with low-glucose DMEM containing $15 \%$ fetal bovine serum. Cells were cultured to the second generation for further transfection or transplantation experiments.

Transfection was performed according to the manufacturer's instructions. Flasks containing $5 \mathrm{ml}$ low-glucose DMEM with $15 \%$ FBS were placed in an incubator at $37^{\circ} \mathrm{C}$ and $5 \% \mathrm{CO}_{2}$. BMMSCs in the exponential growth phase were selected and separated into single-cell suspensions, $5 \times 10^{5}$ cells were centrifuged at $1200 \mathrm{rpm}$ for $5 \mathrm{~min}$, and supernatant was removed completely to produce cell clusters. Then $100 \mu \mathrm{L}$ human MSC nucleofector solution and $2 \mu \mathrm{g}$ recombinant PCDNA 3.1 (-) -SHH eukaryotic expression vector were added to the cell cluster, after careful and gently mixing, the cell cluster was transferred to a special electroporation cup, and high survival program U-23 was selected for transfection. The transfection was completed in a few seconds. The transfected cells were transferred to a pre-buffered medium and gently dispersed. Cells transfected with the same procedure three times were placed in the same culture flask. The flask was placed in an incubator at $37^{\circ} \mathrm{C}$ and $5 \% \mathrm{CO}_{2}$. After $24 \mathrm{~h}$, the medium was changed. Non-adherent cells were discarded and then cultured for another $24 \mathrm{~h}$. Before the transplantation, the transfected cells were extracted and checked by fluorescence photography, and the expression of SHH gene as well as related protein were checked.

\subsection{Reverse Transcription PCR for the Expression of SHH in MSCs}

RNA was extracted by Trizol and cDNA was synthesized by a Reverse Transcription System Kit (Promega). The PCR amplification system was $2 \times$ Taq plus PCR Mix $10 \mu \mathrm{L}$, template DNA $1 \mu \mathrm{L}(80 \mathrm{ng})$, forward primer $0.4 \mathrm{nmol}$, reverse primer $0.4 \mathrm{nmol}$, adding $\mathrm{ddH}_{2} \mathrm{O}$ to terminal volume $20 \mu \mathrm{L}$. The PCR reaction consisted of 4 minutes denaturation at $94^{\circ} \mathrm{C}, 35$ cycles at $94^{\circ} \mathrm{C}$ for 30 seconds, $58^{\circ} \mathrm{C}$ for 30 seconds, $72^{\circ} \mathrm{C}$ for 60 seconds, and 10 minutes prolongation at $72^{\circ} \mathrm{C}$. The forward primer for GAPDH (as reference gene) was CAAGGTCATCCATGACAACTTTG and the reverse primer was GTCCACCACCCTGTTGCTGTAG (Length of Product: $496 \mathrm{bp}$ ). The forward primer for SHH was GGTGGCACCAAGTTAGTGAAGGAT and the reverse primer was AGAAGACCTTCTTGGCACCTTCGT (Length of Product: $130 \mathrm{bp}$ ).

\subsection{Western-Blot for the Expression of SHH in MSCs}

The protein was obtained from MSCs 24 hours after transfection. $10 \mathrm{uL}$ protein sample was then electrophoresed on $10 \%$ SDS-PAGE at constant voltage $110 \mathrm{v}$ and transferred to Nitrocellulose membrane at $66 \mathrm{v}$ for about 2 hours. Subsequently, the membrane was blocked by a blocking buffer for 1 hours, followed by incubating with rabbit anti rat SHH antibody (diluted $1: 250$ ) at $4^{\circ} \mathrm{C}$ overnight. After washed with Tris-buffer saline (TBS) three times, the meberance was incubated with horseradish peroxidase-conjugated secondary antibody (diluted 1:1000) 
at room temperature for 1 hour and washed with TBS again. Finally electrochemiluminescence (ECL) reagent was added. 2 minutes later the film was exposed, developed and fixed.

\subsection{Construction of Myocardial Infarction Model and Cell Transplantation}

A rat model of acute myocardial infarction was generated by coronary artery ligation, and the experimental animals were divided into five groups (40 rats per group, 10 rats per week). Cell transplantation therapy was performed immediately after the establishment of the rat model by direct injection. In transfection group, immediately after the establishment of myocardial infarction model, $5 \times 10^{6}$ BMMSC $^{\mathrm{SHH}}$ were transplanted into the infarct area. The cell only group was transplanted with an equal number of BMMSCs. The gene only group was injected with $200 \mu \mathrm{g}$ pcDNA3.1-Shh plasmid DNA. The mixture group was injected with a mixture of equal number of BMMSCs with $200 \mu \mathrm{g}$ pcDNA3.1-Shh plasmid DNA, and the control group was injected with equal volume of low-glucose cold DMEM. The rats injected with BMMSC ${ }^{\mathrm{SHH}}$ seems more activitive weeks late when compared with others groups.

\subsection{Real Time-PCR}

RNA was extracted with Trizon and reverse transcribed into cDNA. PCR was performed using a $20 \mu \mathrm{L}$ reaction system, the following reagents were added into the PCR reaction tube: $2 \times$ Taq plus PCR Mix $10 \mu \mathrm{L}$, DNA template $1 \mu \mathrm{L}(80 \mathrm{ng})$, upstream and downstream primers $0.4 \mathrm{nmol}$ each. Then ultrapure water was added to a final volume of $20 \mu \mathrm{L}$. The PCR reaction conditions were as follows: $94^{\circ} \mathrm{C}$ for $4 \mathrm{~min}$, followed by 35 cycles of $94^{\circ} \mathrm{C}$ for 30 seconds, $58^{\circ} \mathrm{C}$ for $30 \mathrm{~s}$, and $72^{\circ} \mathrm{C}$ for $1 \mathrm{~min}$. The PCR reaction was ended after a final extension at $72^{\circ} \mathrm{C}$ for $10 \mathrm{~min}$. A $20 \mu \mathrm{L}$ reaction system was also used for $\mathrm{qPCR}$, and the following reagents were added to the PCR reaction tube: SYBR Mix $10 \mu \mathrm{L}$, forward and reverse primers $0.3 \mu \mathrm{mol}$, cDNA $0.5 \mu \mathrm{L}(40$ ng). Then ultrapure water added to $20 \mu \mathrm{L}$. The primer sequences were shown in Table 1. The reaction conditions for qPCR were as follows: $95^{\circ} \mathrm{C}$ for $10 \mathrm{~min}$, followed by 45 cycles of $95^{\circ} \mathrm{C}$ for $15 \mathrm{~s}$ and $60^{\circ} \mathrm{C}$ for $60 \mathrm{~s}$.

\subsection{Statistical Analysis}

The $2^{-\triangle \mathrm{CT}}$ method was used for analysis, $\triangle \mathrm{CT}=$ $\mathrm{CT}^{\text {target gene }}-\mathrm{CT}^{\text {reference gene }}$, and statistical analysis was performed through $2^{-\triangle \mathrm{CT}} \times 1000$. There were 5 groups in this study, each containing 40 samples, 10 of which were harvested per week. Numerical variables were presented as mean $\pm \mathrm{SD}$. In-between group comparison was performed using single-factor analysis of variance (one-way ANOVO) (taking into account that 8 measurements in each group were small sample size, SPSS19 one-way ANOVA was used for overall comparison and pair-wise comparisons were made using Dunnett's T3 test). All calculations were performed using IBM SPSS19. The $\alpha$

Table 1. Primer sequences for real time-PCR.

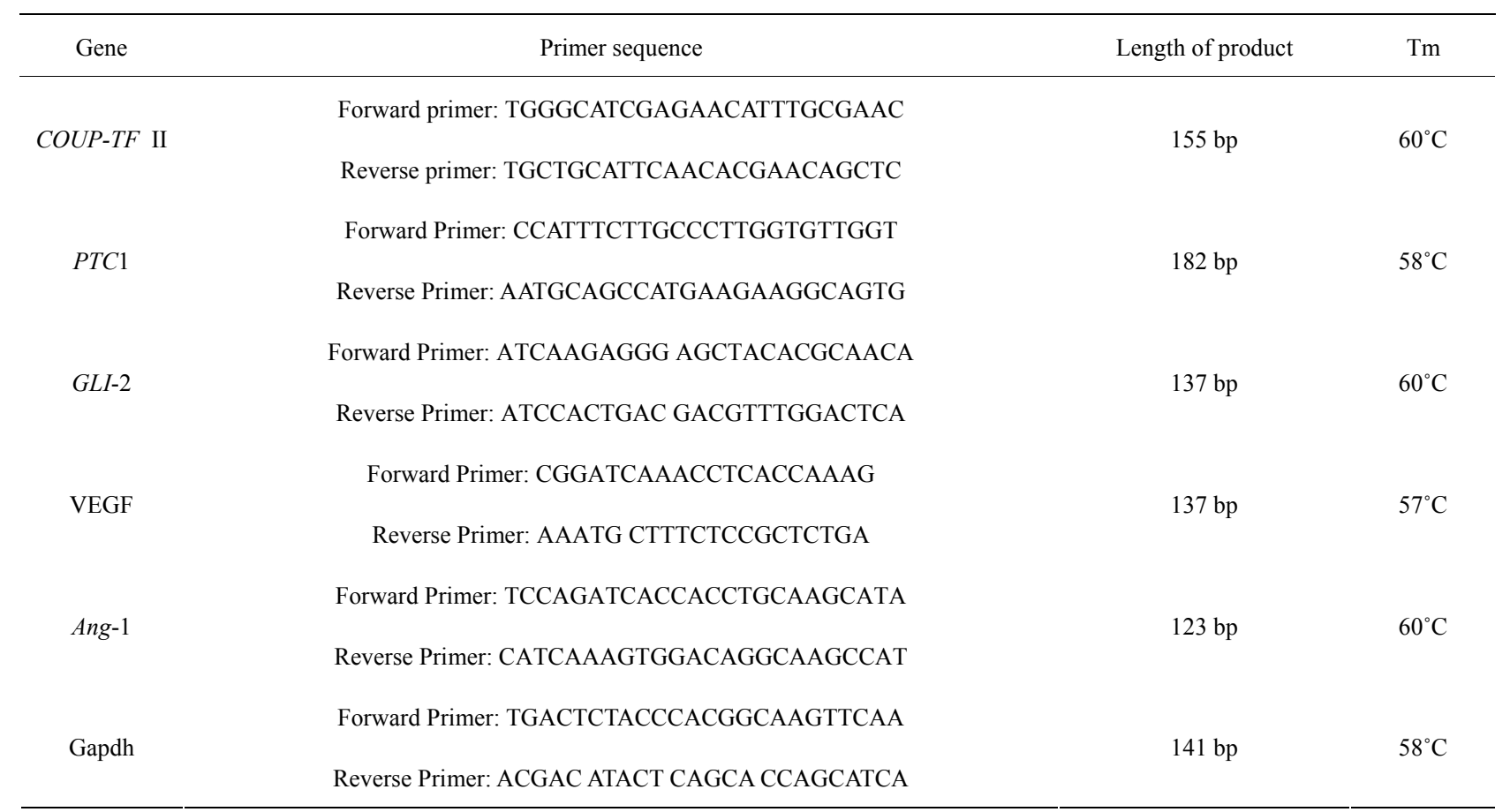


value was set at 0.05 .

\section{RESULTS}

\subsection{Expression of the Targeted Gene after Transfection}

Cells were observed under a fluorescence microscope $48 \mathrm{~h}$ after transfection. As shown in Figure 1, BMMSCs expressing the target genes showed light green fluorescence. PCR data showed that the SHH mRNA expression in BMMSCs transfected with Shh was significantly higher than in non-transfected BMMSCs. Western blot analysis showed that $\mathrm{SHH}$ protein level was significantly higher in BMMSCs transfected with Shh than in nontransfected BMMSCs.

\subsection{Changes of the SHH Downstream Gene Expression at the Transplantation Site}

Seven days after transplantation, the expression of SHH downstream genes, Ptc1, Gli-2, and COUP-TF II was significantly higher than that in the control group, cell only group, gene only group, and mixture group (Ptc1: $P$ $<0.01, P<0.01, P<0.05$, and $P<0.05$, respectively; COUP-TF II: $P<0.01, P<0.01, P<0.05$, and $P<0.05$, respectively; Gli-2: $P<0.01, P<0.01, P<0.05$, and $P<$ 0.05, respectively), as shown in Figure 2. However, tests performed at two weeks, four weeks, and eight weeks after transfection did not show any significant difference

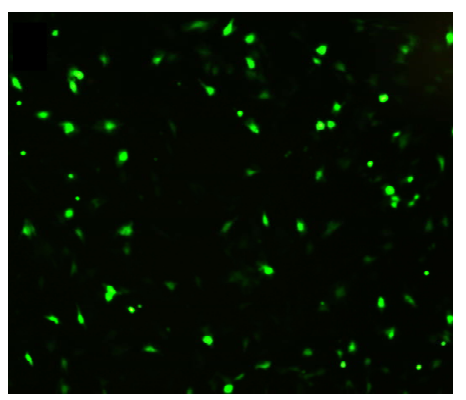

(a)

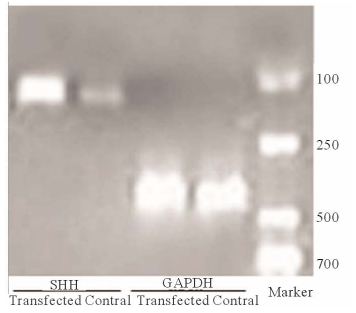

(b)

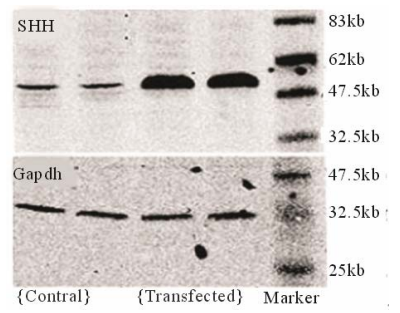

(c)
Figure 1. Target gene expression in transfected cells. (a) Detection of target gene expression after transfection using fluorescence microscopy (labled by green immunofluorescence protein); (b) Detection of target gene expression using PCR; (c) Detection of the expression of the target protein using Western blot analysis.
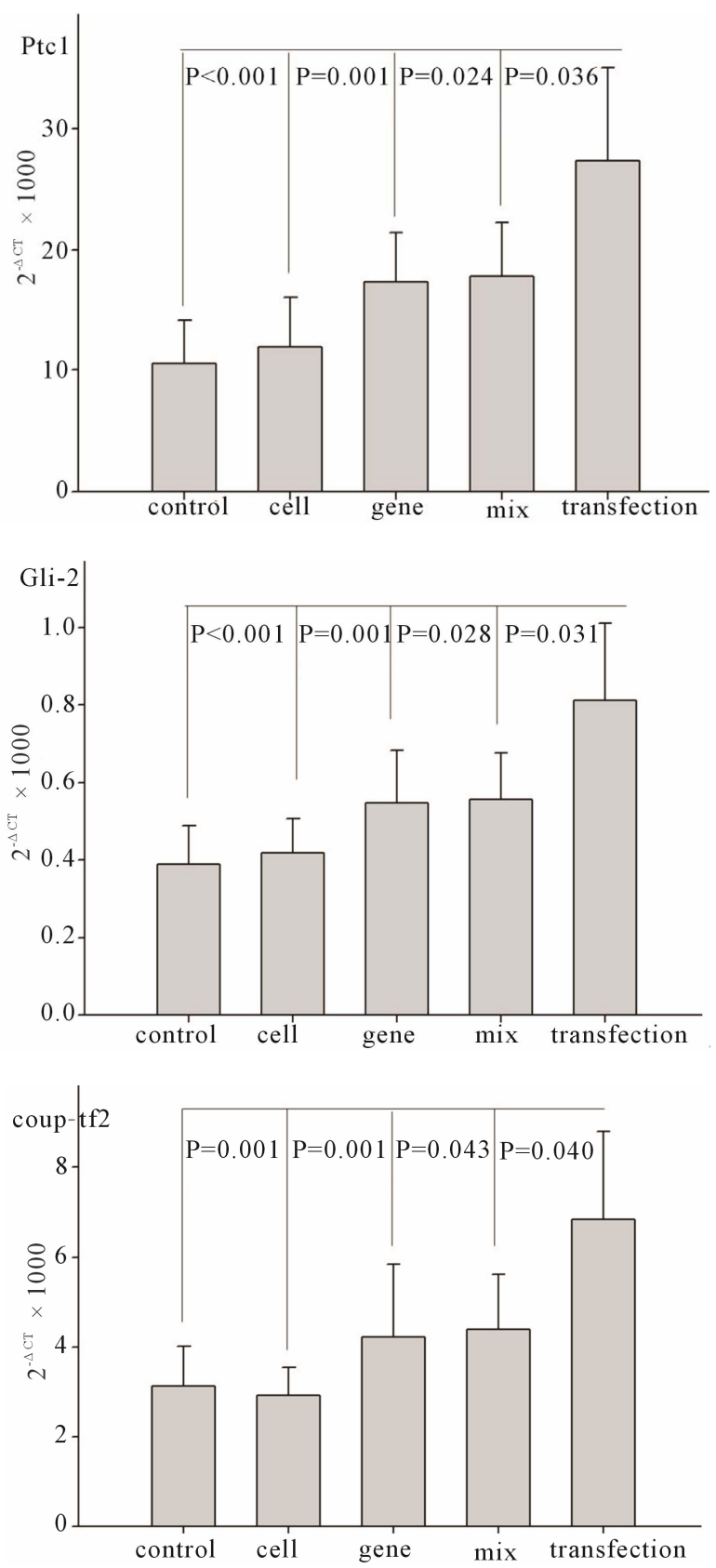

Figure 2. Changes in $\mathrm{SHH}$ downstream gene expression seven days after transplantation. Ptc1: changes in Ptc 1 gene expression among different groups after transplantation. Gli2: changes in Gli-2 gene expression among different groups after transplantation. Coup-TF 2: changes in Coup-TF II gene expression among different groups after transplantation.

in the expression of these genes among different groups $(P>0.05)$.

\subsection{Changes in Levels of Angiogenesis Promoting Factor at the Transplantation Site}

The expression of angiogenesis promoting genes, such 
as Vegf and Ang-1, was significantly upregulated in transfection group than that in the control group, cell only group, and gene only group (Vegf: $P<0.01, P<$ 0.05 , and $P<0.05$, respectively; Ang-1: $P<0.01, P<$ 0.05 , and $P<0.05$ respectively). In the mixture group, expression was slightly but not significantly higher than that in the mixture group (Vegf: $P=0.147$, Ang-1: $P=$ 0.15), as shown in Figure 3. Subsequent tests at two, four, and eight weeks after transplantation showed no significant differences in the levels of expression of these genes $(P>0.05)$.

\section{DISCUSSION}

There have been studies showing that SHH can promote the growth of blood vessels and improve perfusion in the ischemic area, $\mathrm{SHH}$ and its downstream gene were were significantly upregulated in ischemic myocardium and skeletal muscle. This was accompanied by upregulation of the expression of angiogenesis-promoting genes such as Vegf and Ang-1, suggesting that SHH can medi-
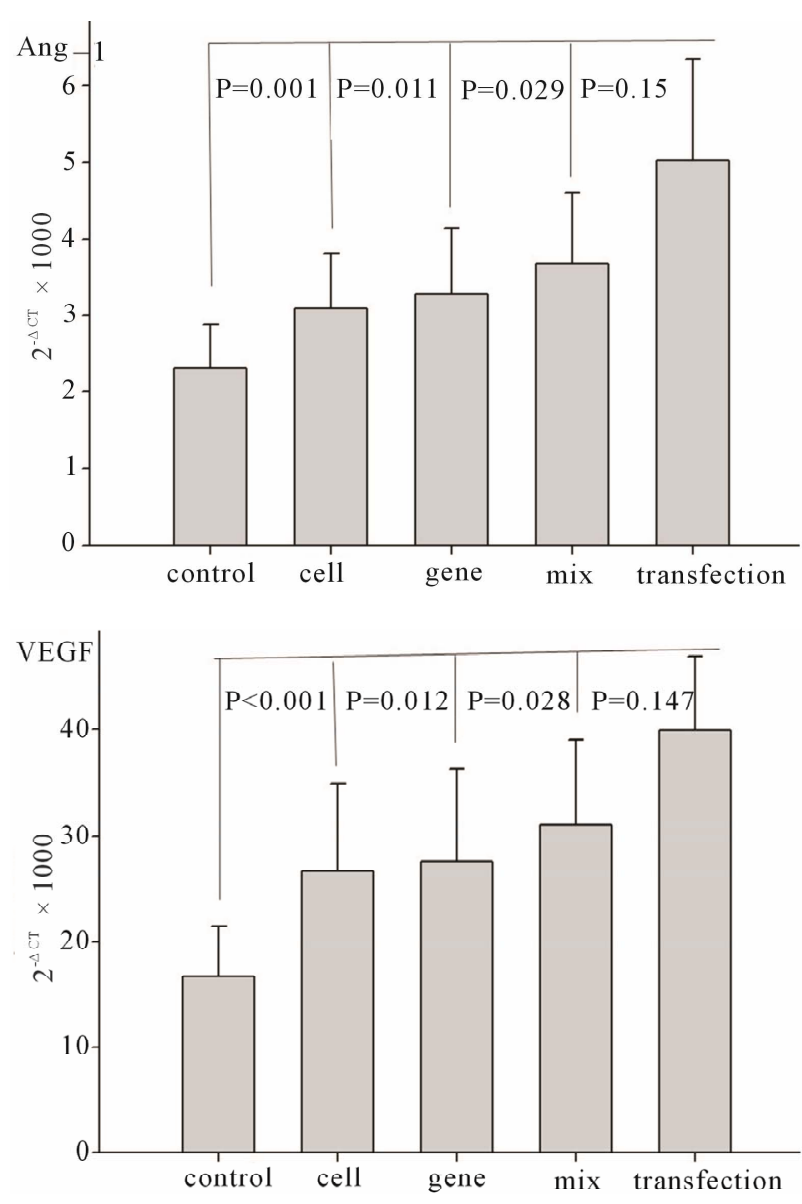

Figure 3. Changes in angiogenic growth factor gene expression seven days after transplantation. Ang-1: changes in Ang-1 gene expression among different groups after transplantation. VEGF: changes in VEGF gene expression among different groups after transplantation. ate a defense reaction in the body, allowing it to resist ischemia and increase blood perfusion. The activation of the SHH signaling pathway is critical to the production of VEGF and other angiogenesis-promoting factors. The topical application of $\mathrm{Sh} h$ or its protein has been shown to increase capillary density and improve blood supply in ischemic hind limb mouse models, cornea, acute myocardial infarction rat models, chronic myocardial infarction pig models, and diabetic neuropathy rat models [4-7].

After being produced and secreted by stromal cells, SHH affects nearby stromal cells in a paracrine manner, causing them to secrete a variety of angiogenic factors, such as VEGF and Ang. At least five important signaling molecules are involved in the SHH-mediated signal transduction pathway [8-11]: 1) Secretory protein SHH. It plays key roles in the formation of the digestive tract, limbs, neural system, cardiovascular, and other organizations. 2) Cell transmembrane protein Patched (Ptc1). This is the receptor for SHH. It has a 12 transmembrane domain structure. 3) Cell transmembrane protein Smoothened (Smo). It has a seven transmembrane domain structure and plays important roles in the $\mathrm{SHH}$ signaling transduction pathway. 4) Zinc finger family transcription factor Gli. It is the downstream signaling molecule of Smoothened and regulates the activity of angiogenic promoting target genes by SHH. 5) COUP-TF II. It is also a downstream signaling molecule of Smoothened. It regulates the activity of $\mathrm{SHH}$ angiogenic promoting target genes by using $\mathrm{SHH}$ as a transcription factor. The signaling pathway is as follows: In the absence of $\mathrm{SHH}$, Ptc1 binds to Smoothened and the activities of Smoothened and Gli are suppressed, resulting in a transcriptional repression state. In the presence of $\mathrm{SHH}$, Ptc 1 binds to its ligand $\mathrm{SHH}$, Smoothened is released and activated, and the Gli gene and COUP-TF II gene are also activated, causing them to become transcriptional activators and bind DNA, inducing the transcription of target genes. Ptcl and Glie are also the target genes of SHH signal in this pathway $[12,13]$. Levels of Ptc1, Gli, and COUP-TF II corresponded to the activity of this signaling pathway. Unlike other factors, SHH cannot cause the migration or proliferation of endothelial cells cultured in vitro. It indirectly promotes the growth of blood vessels by inducing the expression of angiogenic factors, such as VEGF and Ang-1, and others [4,5]. When the SHH-Ptc-Smo signal pathway is activated in receptor cells, the expression of angiogenic target genes such as VEGF and Ang-1 also becomes upregulated.

In this study, we examined the expression of Ptc1, Gli, and COUP-TF II at the transplant site among all groups using real-time quantitative PCR. We further tested the expression of angiogenic growth factors VEGF and Ang-1. Among the specimens obtained during the first 
week, the expression of Ptc1, Gli-2, and COUP-TF II in the transfection group was found to be extremely significantly higher than that of the control group and cell only group $(P<0.01)$. It was also significantly upregulated relative to the gene only group and the mixture group $(P<0.05)$, indicating that the transferred exogenous SHH gene can stimulate the SHH mediated pathway. This effect was most obvious in the transfection group. When the expression of angiogenic growth factors VEGF and Ang-1 was examined, their expression in the transfection group was found to be extremely significantly upregulated relative to the control group $(P<0.01)$ and significantly upregulated relative to the gene only group and cell only group $(P<0.05)$. There was also a slight but not significant trend of upregulation relative to the mixture group (VEGF: $P=0.147$, Ang-1: $P=0.15$ ). These data suggested that both BMMSC and SHH can stimulate angiogenesis and improve local blood perfusion. This effect was most obvious in the transfection group. BMMSC promotes angiogenesis through its ability to stimulate the secretion of angiogenic factors [1415]. We conclude that the transfer of the SHH gene into bone marrow mesenchymal stem cells was successful. After synthesis within the cells, the active part of SHH was secreted and released into the extracellular matrix, producing a paracrine effect. SHH bound to the Ptc1 receptor on the cell membrane of fibroblast cardiomyocytes, which are the most abundant type of cell in the myocardium. This activated the downstream Gli-2 and COUP-TF II signal transduction pathways, regulating the expression of downstream genes, ultimately inducing the upregulation of expression of angiogenic factors such as VEGF and Ang-1, so promoting blood vessel growth. Its therapeutic effect was found to be more pronounced than that of cells alone, genes alone, or cell and gene mixtures (untransfected).

However, starting from the second week, there were no differences in Ptc1, Gli-2, or COUP-TF II expression among all groups. This may be due to the fact that apoptosis of the transplanted cells occurred mainly during the first few days after transplantation. When cells are transplanted into the infarct border zone, because there is still myocardial tissue inflammation, edema, and ischemia in this region, the transplanted cells did not receive sufficient blood. This is worse when transplantation strategy is accompanied by local injection of liquid for lysing cells, causing the transplantation site to swell even more. The changes in the cellular environment made the already damaged cells even more prone to apoptosis. Another important reason is that the transplantation strategy shown in this study cannot target or integrate the SHH DNA fragment into the corresponding gene locus in the chromosomes of BMMSCs. Electric transfection is one form of transient transfection. The plasmids that were not able to target or integrate into specific gene loci in BMMSC chromosomes were metabolized and eliminated. The protein was only transiently expressed and its effects are also transient. After transplantation, although a certain number of cells survived, most of those surviving cells differentiated into cardiomyocytes, which expressed SHH at low levels. More in-depth studies in this area are needed.

\section{ACKNOWLEDGEMENTS}

We appreciate the National Natural Science Foundation of China (No. 30871424), Program for Changjiang Scholars and Innovative Research Team in University (IRT1195) and Hunan Provincial Natural Science Foundation of China (11JJ7005) for their providing funds. We also express our gratitude to all the other doctors and researchers in our hospital attending this research.

\section{REFERENCES}

[1] Siminiak, T. and Kurpisz, M. (2003) Myocardial replacement therapy. Circulation, 108, 1167-1171. doi:10.1161/01.CIR.0000086628.42652.8D

[2] Tomita, S., Li, R.K., Weisel, R.D., Mickle, D.A., Kim, E.J., Sakai, T., et al. (1999) Autologous transplantation of bone marrow cells improves damaged heart function. Circulation, 100, II-247-II-256.

[3] Wang, J.S., Slum-Tim, D., Galipeau, J., Chedrawy, E., Eliopoulos, N. and Chiu, R.C. (2000) Marrow stromal cells for cellular cardiomyoplasty: Feasibility and potential clinical advantages. The Journal of Thoracic and Cardiovascular Surgery, 120, 999-1005. doi: $10.1067 / \mathrm{mtc} .2000 .110250$

[4] Pola, R., Ling, L.E., Aprahamian, T.R., Barban, E., Bosch-Marce, M., Curry, C., et al. (2003) Postnatal recapitulation of embryonic hedgehog pathway in response to skeletal muscle ischemia. Circulation, 108, 479-485. doi:10.1161/01.CIR.0000080338.60981.FA

[5] Pola, R., Ling, L.E., Silver, M., Corbley, M.J., Kearney, M., Blake, P.R., et al. (2001) The morphogen sonic hedgehog is an indirect angiogenic agent upent upregulating two families of angiogenic growth factors. Nature Medicine, 7, 706-711. doi:10.1038/89083

[6] Kusano, K.F., Pola, R., Murayama, T., Curry, C., Kawamoto, A., Iwakura, A., et al. (2005) Sonic hedgehog myocardial gene therapy: Tissue repair through transient reconstitution of embryonic signaling. Nature Medicine, 11, 1197-1204. doi: $10.1038 / \mathrm{nm} 1313$

[7] Kusano, K.F., Allendoerfer, K.L., Munger, W., Pola, R., Bosch-Marce, M., Kirchmair, R., et al. (2004) Sonic hedgehog induces arteriogenesis in diabetic vasa nervorum and restores function in diabetic neuropathy. Arteriosclerosis, Thrombosis, and Vascular Biology, 24, 1-7. doi:10.1161/01.ATV.0000144813.44650.75

[8] Cohen Jr., M. M. (2003) The hedgehog signaling network. American Journal of Medical Genetics, 123, 5-28. doi:10.1002/ajmg.a.20495 
[9] Krishnan, V., Pereira, F.A., Qiu, Y., Chen, C.H., Beachy, P.A., Tsai, S.Y., et al. (1997) Mediation of sonic hedgehog-induced expression of COUP-TF II by a protein phosphatase. Science, 278, 1947-1950.

[10] Sisson, J.C., Ho, K.S., Suyama, K. and Scott, M.P. (1997) Costal2, a novel kinesin-related protein in the hedgehog signaling pathway. Cell, 90, 235-245. doi:10.1016/S0092-8674(00)80332-3

[11] Robbins, D.J., Nybakken, K.E., Kobayashi, R., Sisson, J.C., Bishop, J.M. and Thérond, P.P. (1997) Hedgehog elicits signal transduction by means of a large complex containing the kinesis-related protein costal2. Cell, 90, 225-234. doi:10.1016/S0092-8674(00)80331-1

[12] Marigo, V., Johnson, R.L., Vortkamp, A. and Tabin, C.J. (1996) Sonic hedgehog differentially regulates expression of GLI and GLI3 during limb development. Developmental Biology; 180, 273-283. doi:10.1006/dbio.1996.0300

[13] Marigo, V. and Tabin, C. (1996) Regulation of patched by sonic hedgehog in the developing neural tube. Proceedings of the National Academy of Sciences of the United States of America, 93, 9346-9351. doi:10.1073/pnas.93.18.9346

[14] Kinnaird, T., Stabile, E., Burnett, M.S., Lee, C.W., Barr, S., Fuchs, S., et al. (2004) Marrow-derived stromal cells express genes encoding a broad spectrum of arteriogenic cytokines and promote in vitro and in vivo arteriogenesis through paracrine mechanisms. Circulation Research, 94, 678-685. doi:10.1161/01.RES.0000118601.37875.AC

[15] Ziegelhoeffer, T., Fernandez, B., Kostin, S., Heil, M., Voswinckel, R., Helisch, A., et al. (2004) Bone marrowderived cells do not incorporate into adult growing vasculature. Circulation Research, 94, 230-238. doi:10.1161/01.RES.0000110419.50982.1C 